\title{
COLLATERAL RESPIRATION
}

\section{Spontaneous Reinflation of an Atelectatic Pulmonary Lobule by Collateral Respiration}

\author{
By C. M. VAN ALLEN AND Y. C. SOO
}

(From the Departments of Surgery and Radiology, Peiping Union Medical College, Peiping)

(Received for publication September 14, 1932)

Collateral respiration is a function of the pulmonary lobule whereby the alveoli of that part exchange gases with the outer atmosphere through interlobular connections and the bronchial system supplying neighboring lobules, rather than through the bronchi of the lobule itself $(1,2)$. This occurs when the bronchus of the lobule becomes obstructed while the other passages are open. The nature of the interlobular connections is not entirely understood, but it is definitely known that very minute channels play a part, and it is suspected that these are the pores of Kohn which extend through the septa from alveolus to alveolus. Collateral respiration may be very free; indeed, so free that nearly or quite as much air passes thereby as would pass by direct respiration without bronchial obstruction. Instances are known where nine-tenths of a lobe has suffered bronchial obstruction for indefinitely long periods and remained fully air-containing (3). No such exchange of air has been found between lobes, at least in man, dog and cat; when a whole lobe becomes obstructed the isolation is complete, the absorption of air into the blood stream is not compensated and atelectasis develops.

By virtue of its rôle in maintaining the inflation of obstructed lobules, collateral respiration plays an essential part in the elimination of obstructions from the tertiary and smaller bronchi with coughing; since full inflation of the obstructed portion is necessary to the aerodynamic expulsive action of cough (4). Also, due to collateral respiration obstructive pulmonary emphysema rarely, if ever, occurs in lobular form (5). More important to an understanding of the phase of the subject now to be presented is information concerning the factors in disease which may interfere with collateral respiration (3). This briefly is as follows:

First, the volume of the interlobular exchange that occurs after bronchial obstruction is greatly reduced in shallow breathing, especially if the action of the diaphragm ceases. Nevertheless, even with exceedingly light breathing and complete diaphragmatic immobility, the collateral exchange between obstructed lobules in the lower lobes is enough to maintain the normally air-containing condition. Second, the presence of viscid 
fluids, such as mucus or blood, in those air passages of the free lobules upon which the collateral exchange depends reduces this function; but other hindrances to the exchange being lacking, the amount of such fluids must be very great indeed so to interfere with collateral respiration as to allow atelectasis to develop. The third, and most effective, influence is inflammation of the parenchyma; for it seems that swelling of the alveolar septa develops at the beginning in pneumonia and closes the side channels. Of course, as the infection progresses, the exudate that collects in the alveoli also soon blocks collateral respiration.

An application of this information has been made to explain the comparative frequency of lobular atelectasis in persons recovering from abdominal surgical operations. These patients are not only likely to produce excessive bronchial secretions and to develop bronchiolar obstruction from stagnation of those fluids, but they also tend to use their diaphragms very little in breathing and to develop hypostatic pulmonary edema and pneumonia. Thus, while bronchiolar obstruction in other persons is ordinarily compensated efficiently by collateral respiration, it may not be in these patients.

There remains the problem, whether an atelectatic pulmonary lobule with persistent obstruction of its bronchus can undergo reinflation spontaneously by collateral respiration after the interference with that function disappears. A solution was sought in the experiments to be described.

\section{EXPERIMENTS}

Dogs, which had passed through 2 weeks of quarantine without symptoms of disease, were used. The plan was to obstruct, first, the bronchus of one major pulmonary lobule and, second, the main bronchus of the same lobe: to wait until the whole lobe was atelectatic; to relieve the second obstruction only; and then to see if the lobule with persistent obstruction would become reinflated spontaneously after the rest of the lobe had become air-containing. Ligation was chosen as the method of causing obstruction, after various types of intrabronchial plugs had been tried at length, because it was surer, more selective, and less likely to be followed by serious pulmonary infection. The procedure was carried out in three somewhat different ways:

Method 1. The dog was morphinized, anesthetized with ether, and prepared for positive pressure respiration. The right pleural cavity was opened by incision in the sixth intercostal space, with aseptic technique, and the hilus of the lower lobe was brought to view. The reflection of the lung parenchyma onto the posterolateral aspect of the main bronchus of that lobe was raised for a distance (about $2 \mathrm{~cm}$.) toward the periphery, to the origin of the first bronchial branch of the lobe; and the branch was doubly ligated with silk and divided between the ligatures. Then, after the lungs were inflated completely to exclude pneumothorax, the wound of the chest wall was closed with sutures and the animal was allowed to awaken. Two or three weeks later an x-ray examination was made to satisfy the requirement that the lungs be totally free from consolidation of any sort. Immediately following this, the chest was reopened by the same surgical method as before and the main bronchus of the lobe was freed throughout its circumference at the hilus. A metal pin about $3 \mathrm{~cm}$. long 
was then laid across the left side of the bronchus so that its ends protruded from both front and back, with the head-end in front. A short rubber band was stretched across the right side, with the ends encircling the ends of the pin, tightly compressing and obstructing the bronchus between it and the pin (Fig. 3, insert). A thread was tied to the pin's head and led out of the chest.

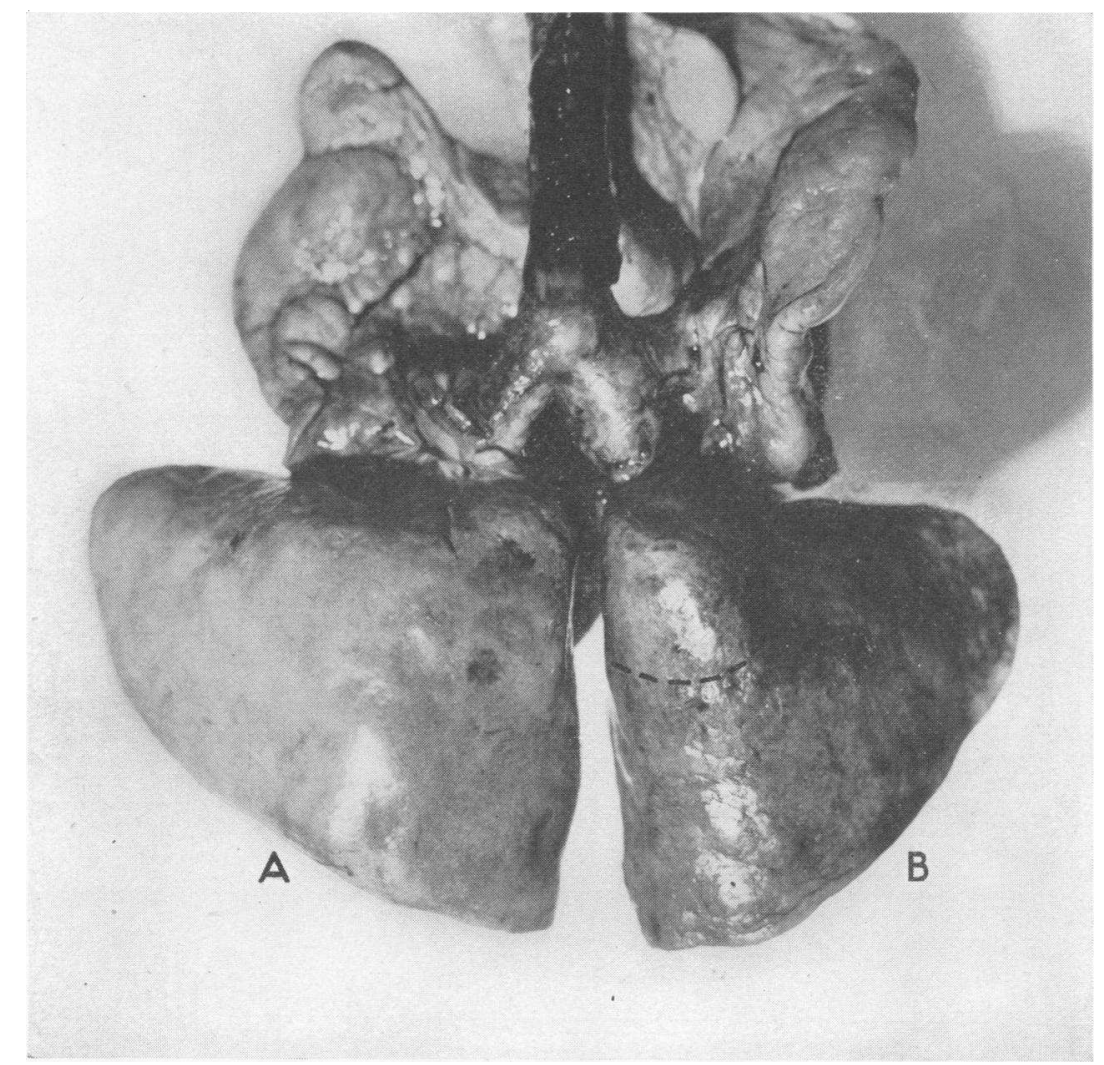

Fig. 1. Photograph of the Lungs of a Dog Treated by Method 2 and Sacrificed 3 Days after Removal of the Lobar Obstruction.

The specimen is arranged with the lower lobes forward for comparison. $A$ : normal lower lobe. $B$ : newly reinflated lobe. The latter is slightly the smaller and is discolored from fibrin on the pleura; the permanently obstructed lobule (outlines indicated approximately by dashes) resembles the rest of the lobe.

After the other lobes were inflated sufficiently to exclude pneumothorax, the chest was closed. The wound with the thread emerging from it was covered with dressings. Twenty-four hours later another $\mathrm{x}$-ray was taken to make certain that the lobe was entirely atelectatic; and then the obstruction of the main bronchus was relieved simply by pulling the thread and pin from the chest. X-ray examinations were made frequently thereafter and, when it appeared that reinflation of the lobe had progressed as far as it would, the dog 
was sacrificed by electrocution. At autopsy, the trachea was first exposed and clamped, the chest was opened, and the lungs were removed in the inflated condition. The specimen was photographed and $x$-rayed, and then subjected to careful gross and microscopic examination.

Method 2. This procedure differed from the first in three points: Both bronchi were obstructed at the same (the only) surgical operation: a thread was attached to the rubber band as well as to the pin, and both objects were withdrawn from the chest; and the time of withdrawal was 48 hours after the application.

Control method. The dog was treated according to method 2 up to a point 24 hours after the operation, when it was sacrificed and examined without removal of the pin and band.

\section{RESLLTS}

Method 1. Two dogs were studied. The roentgenogram taken at the end of that period in which the bronchus of one lobule alone was occluded showed neither atelectasis nor any other sort of pulmonary consolidation. ${ }^{1}$ This finding was verified at the operation that followed, as far as the right lung was concerned. At the end of the period of occlusion of the lobar bronchus, typical roentgen-signs of massive atelectasis of the right lower lobe were found, namely, a ground-glass-like shadow of lobar proportions in the lower part of the right lung-field, sharply demarcated above and inseparably fused medially and below with the shadows of the heart and diaphragm; extensive displacement of the heart toward the lesion; and elevation of the corresponding hemidiaphragm (6). The shadow of the pin could be seen at the hilus. Three days after extraction of the pin, the film showed complete reaeration of the free portion of the right lower lobe but atelectasis of the permanently obstructed lobule. No further change was noted and the animals were sacrificed 5 and 7 weeks later respectively. At autopsy, the lungs were air-containing, save for a few small scattered patches of unresolved pneumonia and an area of atelectasis in the obstructed lobule. Some of the pneumonic foci were situated in the right lower lobe, and one of them, in each dog, lay directly adjacent to the atelectatic tissues. The atelectasis involved all of the obstructed lobule except a few cubic millimeters of the cortex, in one dog, and all but about one-fourth of the cortex, in the other; while the remainder of the lobule in both cases was definitely air-containing. The air-containing portion, in both, was directly adjacent to the non-consolidated tissues of the rest of the lobe. The parenchyma and bronchi of all lobes were distinctly congested and the bronchi contained small quantities of mucopurulent secretion, although these changes were most pronounced in the free part of the right lower lobe. The wall of the bronchus which had been temporarily compressed was swollen to about twice the normal thickness, and on one side of its external surface lay the rubber band embedded deeply

1 The obstructed lobule lay in a part of the lung-field that was plainly visible in the roentgenogram. 
in scar tissue. In the dog which exhibited the greater amount of atelectasis, one end of the band extended through a small hole in the bronchial wall and hung in the lumen. The bronchus of the atelectatic lobule was completely interrupted at its origin, its stumps of amputation were healed shut, and the entire bronchial tree of the lobule distal to that point was distended with glairy viscid mucus.

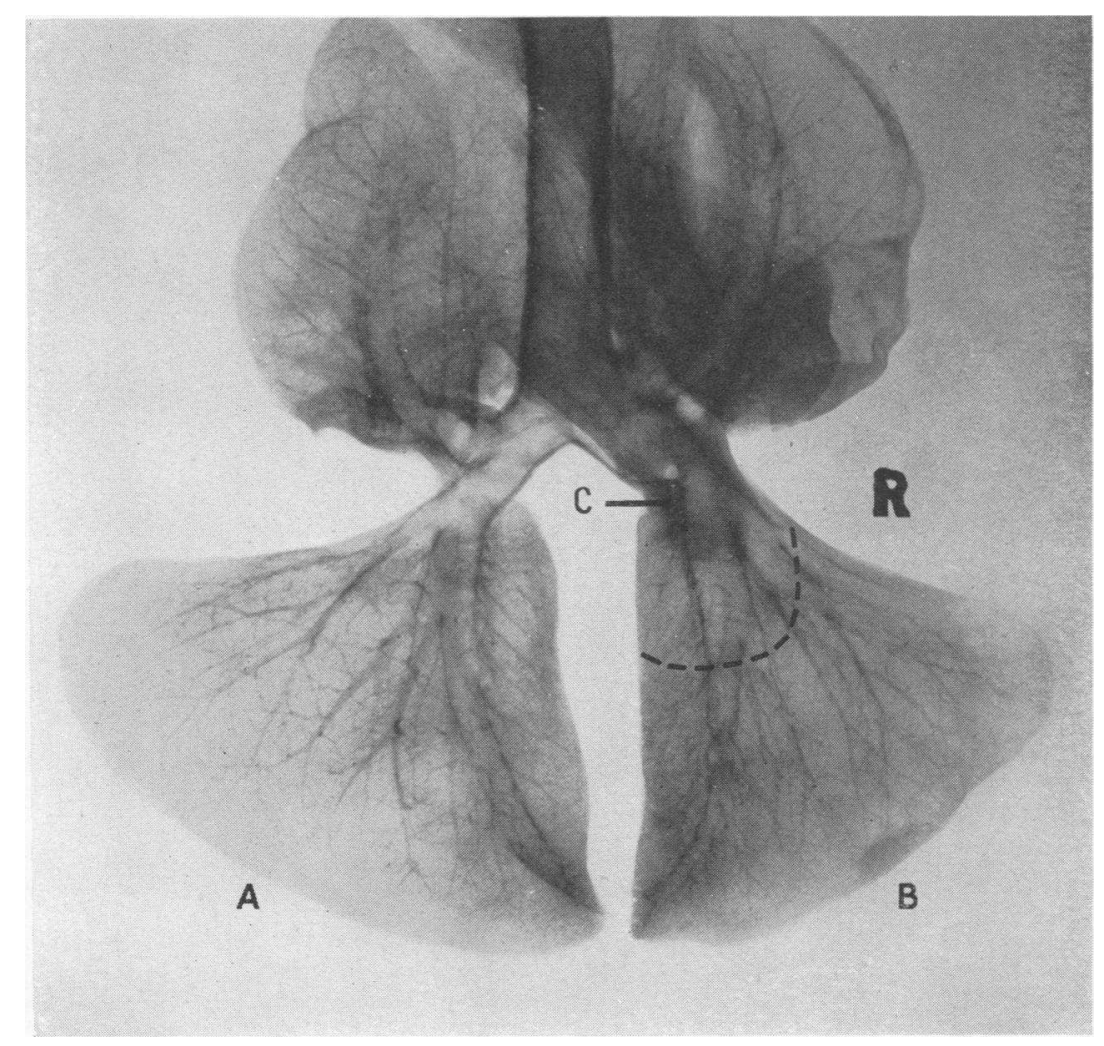

Fig. 2. Roentgenogram of the Specimen of Fig. 1, Demonstrating the Uniformly Air-containing State of the Lungs, Including the Permanently Obstructed Lobule.

At $C$ is the thickening of the bronchial wall where the pin and band had been.

Method 2. The same roentgenographic evidences of massive lobar atelectasis, as already described, were obtained in three dogs just before the removal of the pin and band. Three days afterward in two cases and 9 days afterward in the other, $x$-ray examination showed that reinflation of the entire lobe had taken place. At autopsy, the lungs of the first two dogs appeared normal externally except for the right lower lobe, which 
was slightly smaller than normal ${ }^{2}$ and was somewhat discolored from fibrinous deposits on the pleura (Fig. 1). The permanently obstructed lobule was air-containing throughout, as were also the remainder of the

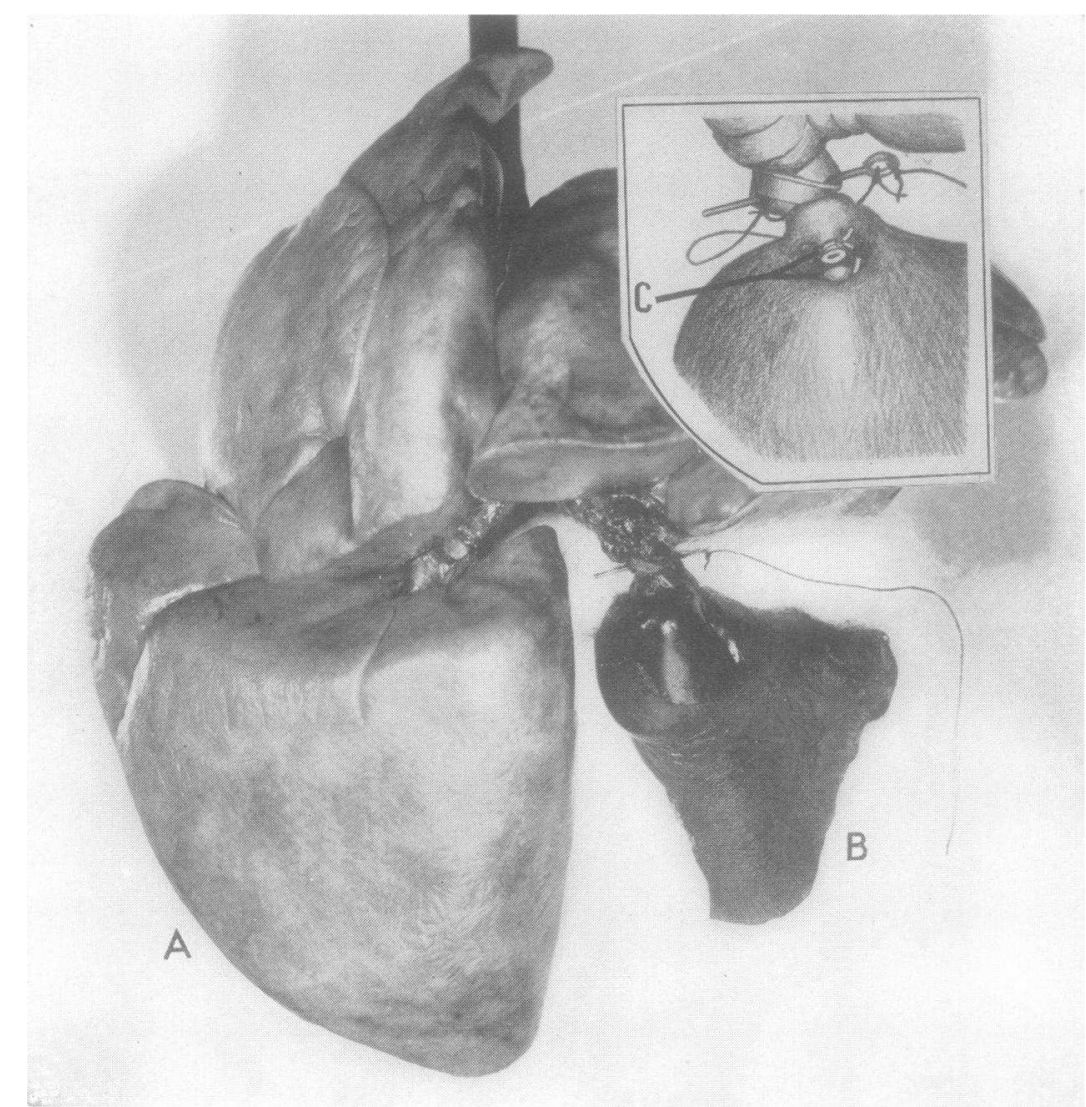

Fig. 3. Photograph of the Lungs of a Dog of the Control Grour, Showing the Atelectasis Produced by the Lobar Obstruction.

A: normal lower lobe. $B$ : atelectatic lobe, with the main bronchus obstructed by pin and band. Insert: diagram of the hilic region of the right lower lobe, showing the arrangement of the pin, band and thread more clearly, as well as the location $(C)$ of the stumps of the ligated and dirided bronchial branch.

2 The lower lobes of a dog's lungs are normally the same size, or very nearly so, according to the measurements of Van Allen and $\mathrm{Wu}_{\mathrm{u}}(7)$. The smaller size of the right one in this case is best explained on the principle demonstrated by the same authors, namely, that an acutely inflamed but non-consolidated part of the lungs is less elastic than the other parts, so that it resists inflation the more and is the smaller when the lungs are inflated in union. In the experiments of these authors, the inflammation was produced by inoculation with pneumococcus, whereas in the present instances it resulted from the trauma of the recent operation and perhaps too from spontaneous infection. Atelectasis was definitely not the cause of the smaller size in either case, since it was lacking. 
right lobe and the other lobes (Fig. 2). There was moderate generalized congestion. The bronchus of the obstructed lobule was completely interrupted and closed at its base; and it and all its branches were filled with mucus. Otherwise the lungs presented no excess of exudate. The autopsy of the third dog, showed essentially similar findings, except that three foci of bronchopneumonia were present in the right upper and middle lobes, the congestion was distinctly greater, and the bronchi of all free parts contained small amounts of inflammatory exudate.

Control method. Two dogs showed the same roentgenographic signs of massive lobar atelectasis just before they were sacrificed as were presented by the others at the time of removal of the lobar obstruction. At autopsy, all lobes were air-containing except the right lower one which was completely atelectatic-very much shrunken, dark reddish grey, and flabby (Fig. 3). The pin and band were in position, completely compressing the bronchus, and the stumps of the amputated bronchial branch were closed. The bronchial tree of the whole lobe contained viscid mucus. There was pronounced generalized pulmonary congestion but no inflammatory consolidation.

\section{CONCLUSIONS}

A major pulmonary lobule with permanently obstructed bronchus may remain completely air-containing while the rest of the lobe remains so, but it becomes atelectatic when the rest of the lobe develops bronchial obstruction and atelectasis. The first effect is probably due to collateral respiration, and the second, to the elimination of that function.

Upon restoration of the main bronchus and reaeration of the parenchyma of the rest of the lobe, the permanently obstructed lobule may become reaerated completely in a few days, probably due to the resumption of collateral respiration.

When the rest of the lobe is inflamed but not consolidated the complete reaeration of the obstructed lobule may be delayed several days; or when the lobe is the seat of bronchopneumonia it may be very incomplete even after several weeks. These behaviors are probably due to the interference offered by such inflammatory states to the collateral passage of air.

The reaeration of the obstructed lobule involves only the parenchyma, while the bronchi remain consolidated with the mucus that starts to accumulate in them soon after the onset of the obstruction. This suggests that, during the reexpansion and subsequent respiration of that lobule, the air passes collaterally throughout the lobule from one to another of the terminal groups of alveoli, perhaps from alveolus to alveolus, without recourse to the bronchial tree. 


\section{COMMENT}

These principles serve to explain certain clinical phenomena. There is, for instance, the observation of the bronchoscopist in postoperative atelectasis, that only one or two of the bronchial branches of an atelectatic lobe need to have the occluding secretions sucked out in order that the entire lobe may become reaerated and the remaining secretions be coughed out in a short time (8). This is indeed a fortunate aspect of the treatment, since not all of the bronchi of a lobe can be reached by endoscopic instruments, and, also, since the plugs in the untreated passages cannot possibly be evacuated by the aerodynamic influence of coughing so long as the parenchyma supplied by those bronchi remains airless. It is a common clinical observation, furthermore, that after reinflation of one part of an atelectatic lobe, the other parts may remain collapsed for many days. These patients exhibit low-grade fever, toxemia, rapid breathing and cough; so the delay in reaeration may well be caused by persisting inflammation and moisture in the parenchyma of the lung, as was the case in some of the experiments just cited.

It is well known that obstructive atelectasis occurs frequently in tuberculosis and may remain permanently after healing of the infection. It is the lobar form that remains, rarely the lobular, although the bronchial obstruction in both forms is often permanent. Bronchiolar occlusions are not hard to find in tuberculous lungs of cadavers by slitting open the small passages leading to the lesions (9). The frequency of such obstructions is not surprising when the fact is recalled that pulmonary tuberculosis commonly begins in the lymphoid tissue that lies in and closely about the bronchiolar walls, especially at the bifurcations (10), and leads to local swelling and necrosis (caseation) and, with healing, to cicatrization. It seems very likely, therefore, that collateral respiration is an important economic factor in extensive pulmonary tuberculosis - in the active stages by maintaining the breathing of obstructed lobules as long as possible, and in the stages of arrest and resolution of the inflammation by reaerating atelectatic and permanently obstructed lobules.

\section{SUMMARY}

Collateral respiration is defined and its various, previously described rôles are briefly reviewed. Experimental evidence is presented to show another function of this property of the lungs: the reinflation of an atelectatic pulmonary lobule when its bronchus is permanently blocked. The evidence indicates, too, that such reinflation may be greatly interfered with when the rest of the same lobe is inflamed. It is suggested that these findings explain certain clinical phenomena associated with re-expansion of atelectatic foci in postoperative atelectasis and in pulmonary tuberculosis. 


\section{BIBLIOGRAPHY}

1. Van Allen, C. M., Lindskog, G. E., and Richter, H. G., Yale J. Biol. and Med., 1930, ii, 297. Gaseous Interchange Between Adjacent Lung Lobules.

2. Van Allen, C. M., Lindskog, G. E., and Richter, H. G., J. Clin. Invest., 1931, x, 559. Collateral Respiration. Transfer of Air Collaterally Between Pulmonary Lobules.

3. Van Allen, C. M., and Jung, T. S., J. Thoracic Surg., 1931, i, 3. Postoperative Atelectasis and Collateral Respiration.

4. Lindskog, G. E., and Van Allen, C. M., Arch. Surg., 1932, xxiv, 204. The Aerodynamics of Bronchial Obstruction.

5. Van Allen, C. M., Surg., Gynec. and Obst. (In Press).

6. Van Allen, C. M., LaField, W. A., and Ross, P. S., Radiology (In Press).

7. Van Allen, C. M., and Wu, C., J. Clin. Invest., 1932, xi, 589. Increased Elastic Tension of the Lung in Experimental Pneumonia.

8. Lee, W. E., Personal communication to the authors.

9. Van Allen, C. M., and Ch'in, K., Unpublished observations.

10. Krause, A. K., Harvey Lect., 1921, xvii, 122. Experimental Studies on Tuberculous Infection. 